\title{
The electronic structure of a shallow acceptor and its bound exciton confined in GaAs/AlGaAs quantum wells
}

\author{
P.O. HOLTZ, Q.X. ZHAO, B. MONEMAR, C. HARRIS, M. SUNDARAM*, J.L. MERZ* and \\ A.C. GOSSARD* \\ Department of Physics and Measurement Technology, Linköping University, 58183 Linköping, Sweden \\ * Center for Quantized Electronic Structures (QUEST), University of California at Santa Barbara, Santa \\ Barbara, CA 93106, U.S.A.
}

\begin{abstract}
The exciton bound (BE) at the shallow Be acceptor confined in a narrow GaAs/AlGaAs quantum well (QW) has been investigated by means of selective photoluminescence (SPL) and PL excitation (PLE) experiments. For the shallow acceptor, several excited states have been observed as two-hole transition (THT) satellites of the BE. The heavy hole $(\mathrm{hh})$-like excited $\mathrm{nS}\left(\Gamma_{6}\right)$ acceptor states dominate the satellite spectrum, but also the light hole (lh)-like excited $2 S\left(\Gamma_{7}\right)$ and the parity forbidden transition to the $2 P_{3 / 2}$ excited state have been monitored in SPL spectra. When detecting a THT satellite, the $1 \mathrm{~S}\left(\Gamma_{6}\right)$ hh-like acceptor ground state has been spectrally resolved from the $1 S\left(\Gamma_{7}\right)$ lh-like state in PLE spectra. Several BE states have been theoretically predicted and observed in PLE spectra with the $\mathrm{J}=5 / 2$ state at lowest energy, like in bulk GaAs. The proposed interpretation of the acceptor hh- and lh-states is confirmed by SPL and PLE experiments in the presence of an applied magnetic field and in polarized PLE measurements. An effective g-value for the acceptor BE recombination in varying degree of confinement is derived from these Zeeman measurements.
\end{abstract}

\section{INTRODUCTION}

The upper valence band is fourfold degenerate near the $\Gamma$ point in the Brillouin zone of bulk GaAs, if the spin degeneracy and the spin-orbit interaction is taken into account. In the case of the reduced symmetry of a two-dimensional GaAs/AlGaAs quantum well (QW), the $\mathrm{QW}$ potential lifts this degeneracy of the valence band also at $k=0$. Two Kramers doublets are formed, characterized by the $m$ quantum numbers: The $m_{j}= \pm 3 / 2$ heavy hole (hh) and the $m_{j}= \pm 1 / 2$ light hole (lh) states.

For the case of an acceptor, the symmetry is reduced, when going from bulk to a QW: From $T_{d}$ for a substitutional acceptor in bulk to $D_{2 d}$ for an acceptor in the center of a QW. For the case of a 2D acceptor, a complicated mixing between different states at finite $\mathrm{k}$ has to be taken into account. Theoretical approaches on the 2D acceptor states have been reported, based on either the effective mass theory [1] or the effective tight binding model [2]. Both approaches result in an acceptor ground state, which splits into two doublet states: A more strongly bound state of dominating hh character and another state of mainly lh character. Although we will hereafter denote these states as the hh and lh acceptor states, it should be kept in mind that this assignment to particular subbands is less appropriate for the acceptor states. An assignment to particular subbands is in principle only applicable for the excited acceptor states, for which the Coulomb coupling between the subbands constitutes only a correction term [3]. For the acceptor ground states, on the other hand, the binding energies are comparable to or even exceed the subband separation and these states are in fact strongly mixed. The predicted splitting between the $\mathrm{hh}$ and $\mathrm{lh}$ acceptor ground states is significantly reduced compared to the corresponding subband separation. E.g. for a central acceptor in a $150 \AA$ wide $Q W$, calculations by S. Fraizzoli and A. Pasquarello [1] result in an energy splitting of about $0.5 \mathrm{meV}$ between the hh and h acceptor ground states to be compared with a hh and lh subband separation of about $7 \mathrm{meV}$ for the same well width. 


\section{THE BOUND EXCITON}

The acceptor might bind an exciton (BE) in a three electronic particle system, two holes and one electron. Due to the complexity of this system, no theoretical calculation on the energy levels of the acceptor BE in a QW has been reported so far. It is outside the scope of this work to present a detailed prediction for the $\mathrm{BE}$ energy levels and we here rather extend existing information for the acceptor $\mathrm{BE}$ in bulk GaAs to the corresponding 2D case. To start with the bulk, we know that $\mathrm{j}$-j coupling of the two bound holes involved gives rise to two states, $\mathrm{J}=0$ and $\mathrm{J}=2$. The energy separation between the $\mathrm{J}=0$ and $\mathrm{J}=2$ states is determined by the hole-hole interaction. If also the interaction with the electron is taken into account, three states are formed in case of bulk GaAs, the $\mathrm{J}=1 / 2,3 / 2$ and $5 / 2$ states with the $\mathrm{J}=5 / 2$ state at the lowest energy for shallow acceptors in GaAs [4]. If we now turn to the BE in the 2D case, the QW confinement effect is included in our calculations via the introduction of an axial crystal field perturbation. For a proper theoretical treatment, the interaction between the three electronic particles has been taken into account by using an effective perturbation spin-Hamiltonian consisting of three terms, which describe the hole-electron and hole-hole interaction and the crystal field effect [5]. The resulting electronic structure is shown in Fig. 1.

\section{EXPERIMENTAL}

The samples used in this study were grown by MBE on semi-insulating (100) GaAs substrates under $\mathrm{As}_{4}$-rich conditions. The samples were grown at a temperature of nominally $680^{\circ} \mathrm{C}$ and without interruptions at the QW interfaces. On top of the substrate, a $0.35 \mu \mathrm{m}$ undoped GaAs buffer layer was grown. The structures investigated contained multiple QW structures with 50 periods of alternating layers of GaAs QWs with $85 \AA<\mathrm{L}_{\mathrm{z}}<150 \AA$ and $150 \AA$ wide $\mathrm{Al}_{0.30} \mathrm{Ga}_{0.70}$ As barriers. The QW structures were selectively doped with Be acceptors in the central $20 \%$ of the QWs. The Be-concentration has been varied in a wide range, but the spectra shown in this paper are measured on samples with a concentration of $3 \times 10^{16}$ to $1 \times 10^{17} \mathrm{~cm}^{-3}$.

For the selective PL (SPL) and PL excitation (PLE) measurements an $\mathrm{Ar}^{+}$ion laser was used to pump a Titanium doped Sapphire solid-state laser. The emitted light from the samples, perpendicular to the incident laser beam, was focused on the slits of a $1 \mathrm{~m}$ double-grating monochromator and detected with a dry-ice-cooled GaAs photomultiplier. The magneto-optical measurements have been performed in magnetic fields up to $16 \mathrm{~T}$ applied perpendicular to the $\mathrm{QW}$ layers. The excitation light from the laser is coupled onto the sample via an optical fiber. The same optical fiber is used for the emission light from the sample onto the monochromator.

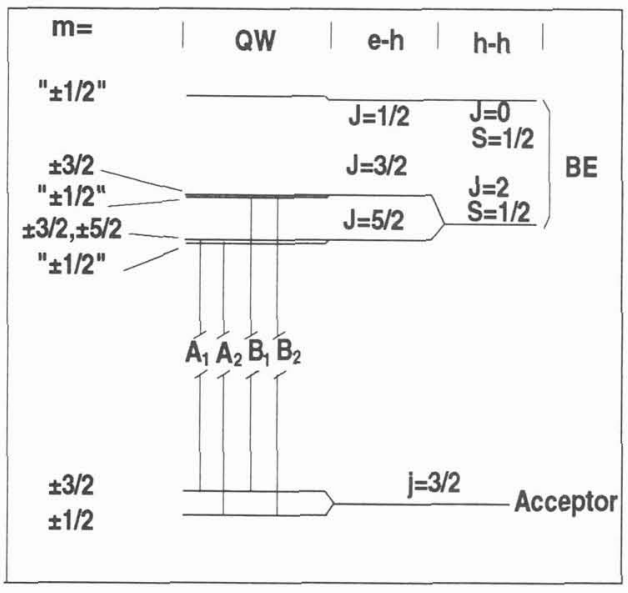

Fig. 1 The electronic structure for the acceptor ground state and the acceptor $B E$ in a $100 \AA$ wide $Q W$, together with the transitions observed in PLE spectra. The BE states are denoted by " $\mathrm{m}$ " when $\mathrm{m}$ is not a good quantum number, since these states are strongly perturbed by the QW potential.

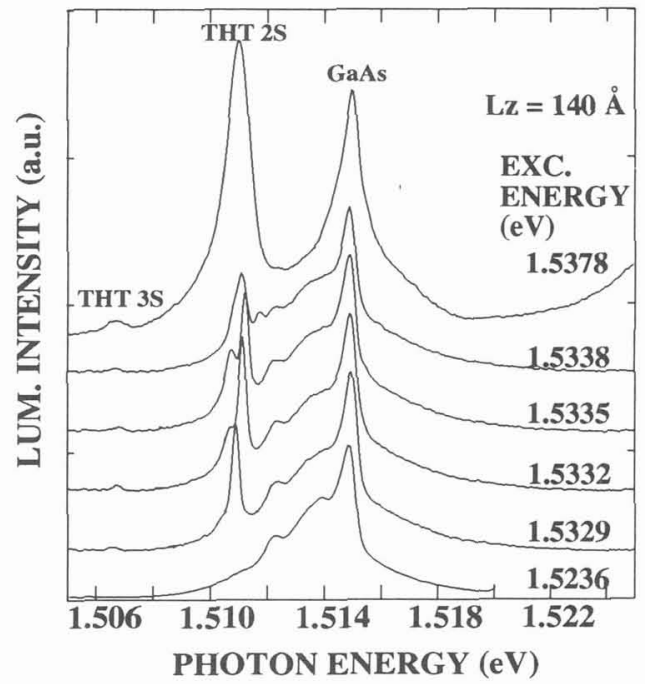

Fig. 2 SPL spectra for the $140 \AA$ wide QW. In the upper spectrum, the excitation energy is resonant with the FE hh state. The bottom spectrum shows the GaAs related emissions with the excitation energy well below the QW energy, while the remaining spectra are measured with the excitation close to or resonant with the BE. 


\section{ZERO-FIELD SPECTROSCOPIC RESULTS}

In addition to the dominating free exciton (FE) and acceptor BE peaks in PL spectra, satellite peaks due to two-hole transitions (THTs) appear at lower energy [6]. The THT satellites can be significantly enhanced by excitation resonant with the FE. In case of excitation close to or resonant with the acceptor BE, a satellite originating from a Resonant Raman Scattering (RRS) process appears in the spectrum [6]. An example of a satellite spectrum with excitation in the excitonic range, is shown in Fig. 2 for the case of a $140 \AA$ wide QW doped to a level of $5 \times 10^{16} \mathrm{~cm}^{-3}$ in the central $20 \%$ of the QW. This SPL spectrum exhibits satellites corresponding to acceptor excitations to the $2 S\left(\Gamma_{6}\right)$ and $3 S\left(\Gamma_{6}\right)$ states. The THT satellites are as strongest when the excitation is resonant with the hh FE as observed in PLE. As the excitation is downshifted to be resonant with the BE PL peak, the position of the satellite peaks shift with the excitation energy in agreement with resonant Raman scattering (RRS) description [6]. Also, since the satellite linewidth is reduced, it is possible to resolve a doublet structure. It is important to point out that the intensity ratio between the two RRS components depends on the excitation photon energy (Fig. 2), which is consistent with the selective excitation description. In addition to the information about the acceptor excited states, the observation of the THT satellites opens the possibility to monitor the BE in PLE spectra, i.e. in the absorption mode.

With the detection energy close to any of the THT peaks, the BE appears in the PLE spectrum in addition to the usually observed FE peaks as illustrated in Fig. 3 for a $100 \AA$ wide $Q W$. An interesting multiline structure of the acceptor $\mathrm{BE}$ (denoted $\mathrm{A}_{1}, \mathrm{~A}_{2}$ and $\mathrm{B}_{1}$ in Fig. 3 ) is then observed. The energy separation between the strongest $A_{1}$ and $B_{1}$ components is $1.7 \mathrm{meV}$ for a $100 \AA$ wide $Q W$. The intensity ratio $A_{1} / B_{1}$ remains almost constant with increasing temperature (Fig. 3), i.e. no thermalization between these components, which is consistent with a splitting in the final state, the BE state, in an absorption process. The $A_{1}-B_{1}$ separation is interpreted as the splitting between the $J=5 / 2$ and $J=3 / 2 \mathrm{BE}$ states (see Fig. 1). The next excited $\mathrm{J}=1 / 2 \mathrm{BE}$ state is predicted to be upshifted about $4 \mathrm{meV}$ versus the $\mathrm{J}=5 / 2$ state (Fig. 1), i.e. it is close to the FE hh peak position and has not been possible to observe in PLE spectra.

The splitting between the $A_{1}$ and $A_{2}$ components measures to $0.65 \mathrm{meV}$ for the $100 \AA$ wide $Q W$ and is found to decrease with increasing $Q W$ width. The $A_{1}$ and $A_{2}$ components exhibit, in contrast to the $A_{1}$

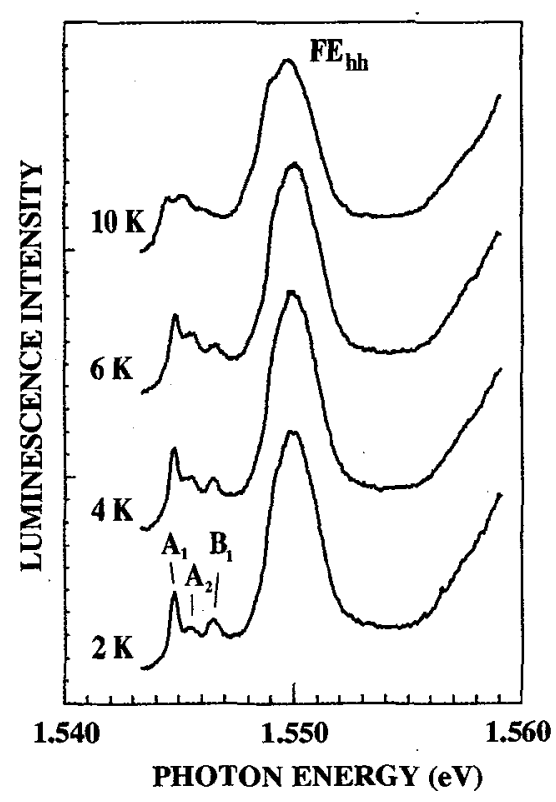

Fig. 3 PLE spectra of a $100 \AA$ wide QW measured at some different temperatures with the detection resonant with the $2 S$ THT satellite.

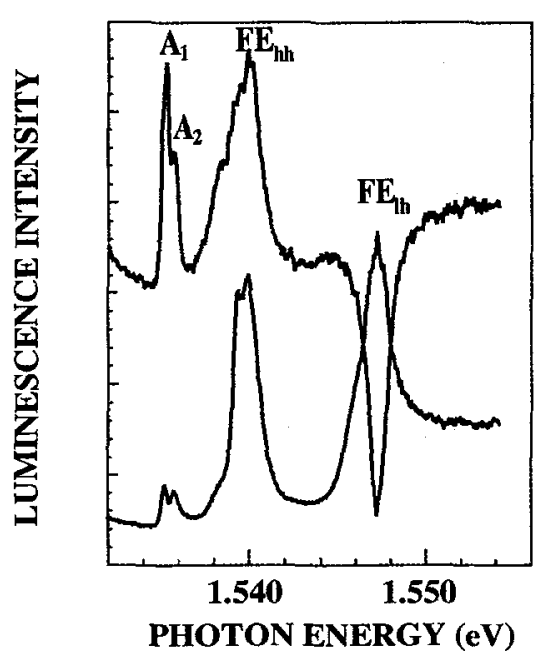

Fig. 4 The polarized PLE spectrum (the upper spectrum) of the $140 \AA$ wide QW in comparison with the unpolarized reference spectrum (the lower spectrum) 
and $B_{1}$ components, a pronounced thermalization behavior (Fig. 3), consistent with a splitting originating from the initial state in absorption, i.e. between the $1 \mathrm{~S}\left(\Gamma_{6}\right) \mathrm{hh}$ and $1 \mathrm{~S}\left(\Gamma_{7}\right) \mathrm{lh}$ acceptor states. This interpretation is confirmed by polarized PLE measurements (Fig. 4). The differential signal between the left-hand and right-hand circularly polarized recombination light from the sample, when the $2 S$ THT satellite is detected, is shown in the upper spectrum together with the corresponding unpolarized PLE reference spectrum below. For the FE states, the hh FE exhibits a positive differential signal in the polarized PLE spectrum, while the $\mathrm{lh}$ FE shows a correspondingly negative signal (Fig. 4). For the case of the $\mathrm{BE}$ components, the $\mathrm{A}_{1}$ peak exhibits a positive differential output signal in the polarized PLE spectrum similarly to the hh FE (Fig. 4). However, also the $A_{2}$ peak, interpreted as due to the acceptor lh state, exhibits a positive signal although at a significantly reduced intensity level in comparison with the unpolarized PLE reference spectrum. An intensity ratio different from the corresponding hh and $\mathrm{lh} F E$ states can be understood by taking the considerable mixing of acceptor states into account. In fact, the observed $\mathrm{A}_{1} / \mathrm{A}_{2}$ intensity ratio could give a hint about the hh-lh mixing rate for the acceptor states.

\section{MAGNETO-OPTICAL RESULTS}

In the presence of a magnetic field, the degeneracy of the acceptor ground state as well as the BE state is expected to be lifted. This is clearly observed in the PL spectrum with a magnetic field applied perpendicular to the $\mathrm{QW}$ layers, in which a splitting of the BE peak into two components is observed for fields higher than $\approx 10 \mathrm{~T}$. From this linear Zeeman splitting of the BE peak, an effective g-value, geff, is derived. The so-obtained effective g-value, geff, is a combination of the splitting in the initial state, the BE state, and the splitting in the final state of the emission, i.e. the $m_{j}= \pm 3 / 2$ acceptor states. The evaluated effective g-value is found to be strongly dependent on the degree of confinement: From geff $=0.66$ for the shallow acceptor BE recombination in bulk GaAs to $g_{\text {eff }}=1.52$ for the $\mathrm{BE}$ recombination in a $150 \AA$ wide $\mathrm{QW}$ and is further increased to geff $=1.89$ in a $100 \AA$ wide $\mathrm{QW}$. The two components of the acceptor $\mathrm{BE}$ observed in the presence of a magnetic field are strongly thermalized as expected for a transition with splitting in the initial state in emission, the BE state. However, it is notable that it is the low energy component, which gains intensity with increasing temperature in this case. Such a thermal behavior is not consistent with the $J=1 / 2$ state as the lowest $B E$ state. This conclusion is based on the following facts: The main part of the combined observed splitting originates from the final state, the acceptor state, since $\left(\mathrm{g}_{\mathrm{h}} \mid\right.$ $>\left|g_{\mathrm{e}}\right|$. Furthermore, we have the transition selection rule $\operatorname{lm}= \pm 1 / 2>\leftrightarrow \operatorname{lm}= \pm 3 / 2>$. Finally, the hole $g$ value is positive, $g_{h}>0$. These facts are not consistent with a negative electron $g$-value, as expected for the $\mathrm{J}=1 / 2$ state assuming that the electron $\mathrm{g}$-value, $\mathrm{g}_{\mathrm{e}}=-0.4 \mathrm{in}$ bulk, remains negative also in $\mathrm{QWs}$. We are then left with the same order of BE states as concluded for shallow acceptors in GaAs [4], i.e. with the $J=$ $5 / 2$ BE state lowest.

From the quadratic Zeeman shift, the diamagnetic shift, we can evaluate the hydrogenic orbital radius from second order perturbation theory of the acceptor $B E[7]$. The diamagnetic shift rate observed for the acceptor BE recombination in PL decreases from $2.8 \times 10^{-2} \mathrm{meV}^{2} \mathrm{~T}^{2}$ in the bulk case to $2.1 \times 10^{-2}$ meV/T $T^{2}$ for the $150 \AA$ wide $\mathrm{QW}$. This reduction of the diamagnetic shift rate corresponds to a reduction of the hydrogenic orbital radius by $13 \%$ for the acceptor $\mathrm{BE}$, when going from bulk GaAs to a $150 \AA$ wide GaAs/AlGaAs QW.

\section{SUMMARY}

The following is a summary of the major results obtained:

1. The hh/lh splitting of the acceptor ground state is observed in PLE and SPL and is found to be in good agreement with theoretical predictions.

2. A simple model for the electronic structure of the acceptor $B E$ has been evaluated and is compared with experimental results. In addition to the lowest $J=5 / 2$ state, the higher $J=3 / 2$ state is also resolved in PLE spectra.

3. The effective $g$-value of the acceptor $\mathrm{BE}$ recombination is determined as a function of confinement.

4. The hydrogenic orbital radius estimated from the diamagnetic shift is found to be strongly reduced from bulk to the QW case.

\section{REFERENCES}

1. See e.g. S. Fraizzoli and A. Pasquarello, Phys. Rev. B44, 1118 (1991)

2. G.T. Einevoll andY.C.Chang, Phys. Rev. B41, 1447 (1990)

3. A. Pasquarello, L.C. Andreani, and R. Buczko, Phys. Rev. B40, 5602 (1989)

4. A.M. White, P.J. Dean and B. Day, J. Phys. C, Vol. 7, 1400 (1974)

5. P.O. Holtz, Q.X. Zhao, B. Monemar, M. Sundaram, J.L. Merz, A.C. Gossard, Phys. Rev. B, in press

6. P.O. Holtz, K. Doughty, M. Sundaram, J.L. Merz, and A.C. Gossard, Phys. Rev. B40, 12338 (1989)

7. W. Rühle and D. Bimberg, Phys. Rev. B12, 2382 (1975) 